\title{
Design and Development of Adaptive Fuzzy Control System for Power Management in Residential Smart Grid Using Bat Algorithm
}

\author{
Venkatachalam Viknesh $^{1}$ (D) . Venugopal Manikandan ${ }^{1}$
}

Received: 17 May 2018 / Accepted: 13 November 2018 / Published online: 22 November 2018

(C) Springer Nature Singapore Pte Ltd. 2018

\begin{abstract}
An adaptive fuzzy logic control system was designed for the management of power in smart grid. The system was developed using BAT algorithm. This paper analyses the approach of adaptive fuzzy logic system accompanied with bat algorithm in power management of smart grid. The path of carrying information i.e. communication layer between users and the energy provider in smart grid is analyzed. To achieve this, behavior of bats and its algorithm is studied and compared with vectors in adaptive fuzzy logic system and applied in power utility side of smart grid. It helps in indicating peak in load and also helps in prediction of power consumption in users. The offline algorithm is developed, simulated using Matlab 16a and simulation results are evaluated.
\end{abstract}

Keywords Fuzzy logic control system $\cdot$ Power management system $\cdot$ Smart grid $\cdot$ BAT algorithm

\section{Introduction}

At present power grids are designed to operate as consolidated structure which consists of generation, transmission, and distribution of electric power. It also supported with control devices to maintain its stability, reliability and efficiency [1]. The next development in grids must have smart facilities of communication among the user and energy provider, real time management of the sources in the grid, and the algorithmic approach for power management techniques. The two main key differences between normal grid and smart grid are consumer participation and accommodation of renewable energy generation and storage. Consumer participation is must whether the user is involved in either demand response or distribution energy resource technique. Demand side management is one of the most important problems in smart grid research, which aims to match electricity demand to supply for enhanced energy efficiency and

Venkatachalam Viknesh

viknesh.tv@cit.edu.in

Venugopal Manikandan manikandan@cit.edu.in

1 Department of electrical and electronics engineering, Coimbatore Institute of Technology, Coimbatore 641014, India demand profile while considering user utility cost and price. Smart grid is involving the renewable source as plug and play basis. Smart grid is a large system of systems where each domain consists of power layer, energy layer, communication layer and information \& technology (IT) enabled layer. Communication and IT layer is emerging topic in smart infrastructure. The success of the smart grid mostly depends on the wide-spread participation of users. The component of communication is somewhat lacking behind in many such smart grids. This is the focusing aspects of many researchers in including this layer in the smart grid [2]. Researchers have been focusing on peak shift or peak reduction for reducing the grid deployment and operational cost, as well as on reducing user or energy provider's cost. This motivated to develop a novel meta heuristic algorithm based fuzzy logic control system for the power management in the smart grid. Two way energy and information flows in the smart grids together with the smart devices, bring new perspectives to energy management and demand response. In this work one way energy and two way information flow were adopted in the residential type smart grid as shown in Fig. 1.

\section{Bat Algorithm}

As we know, bat has unique abilities such as finding their food and recognizing different types of insects even in a complete darkness [4]. The basic principle is echolocation 
Fig. 1 Distributed energy generation

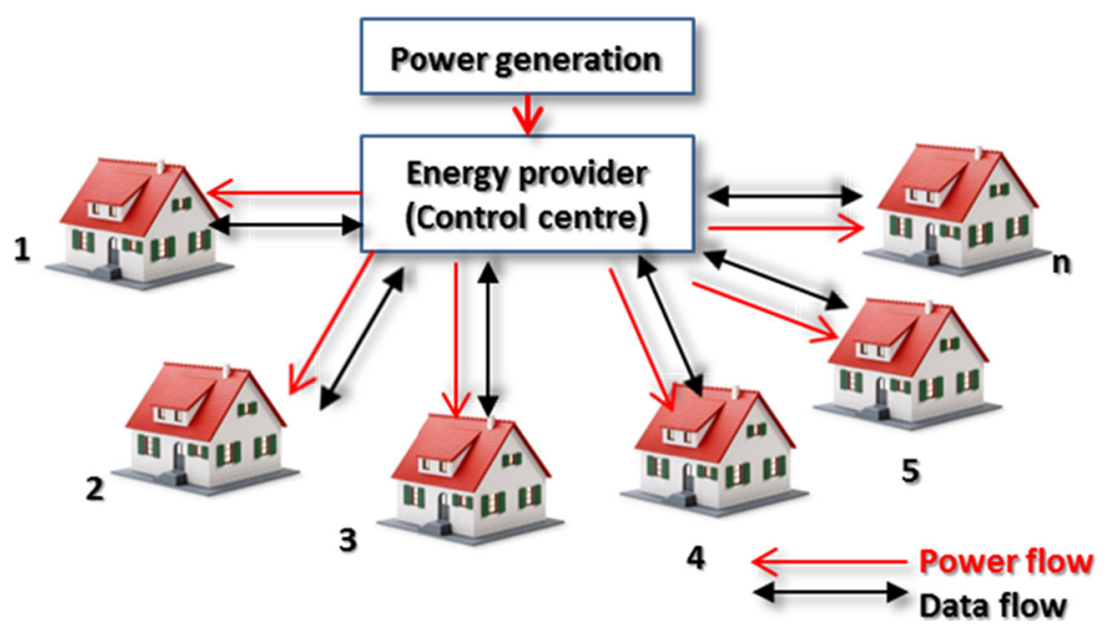

technique that is by emitting high frequency audio signals and sensing the intensity and strength of the reflected signal [5]. Three idealized rules as in Meta heuristic algorithm are executed by the bat during the short span of its transit.

The rules are given as follows.

i. All bats produce ultrasonic sound and receive the echo from the obstacles in their path.

ii. Bats fly randomly with velocity at position with a fixed frequency, varying wavelength and loudness to search for prey. They can automatically adjust the frequency of their emitted pulses and adjust the rate of pulse emission

iii. Although the loudness can vary in many ways, it is assumed that the loudness varies from a positive maximum to a minimum constant value.

The above rules can be mathematically modeled as follows.

The movement of the real bat is assumed as virtual bat in this model. Its maximum loudness and minimum loudness are assumed as $A_{\max }$ and $A_{\text {min }}$ respectively [3]. At any instant of time its position, velocity, and frequency are represented as $x_{i}, v_{i}$ and $f_{i}$ respectively. Let $f_{\min }$ and $f_{\max }$ are its maximum and minimum frequencies of ultrasonic sound. The instantaneous frequency $f_{i}$ can be written as

$\mathrm{f}_{\mathrm{i}}=\mathrm{f}_{\min }+\left(\mathrm{f}_{\max }-\mathrm{f}_{\min }\right) \quad \beta$

Where $\beta \epsilon[0,1]$ is a random vector with uniform distribution.

The rate of emission of the sound $r \in[0,1]$ which can adjust depending on the target and it is updated emission rate can be written as

$r_{i}(t+1)=r_{i}\left(A_{i}\right)\left(1-e^{-\gamma t}\right)$

Where $A_{i}$ is the loudness of the sound

Similarly the updated position and velocity can be written as $\mathrm{x}_{\mathrm{i}}^{\mathrm{t}}$ and $\mathrm{v}_{\mathrm{i}}^{\mathrm{t}}$ respectively.

$v_{i}^{t}=v_{i}^{t-1}+\left(x_{i}^{t-1}-X_{n e w}\right) f_{i}$ $x_{i}^{t}=x_{i}^{t-1}+v_{i}^{t}$

$\mathrm{x}_{\text {new }}=\mathrm{x}_{\text {old }}+£ \mathrm{~A}^{\mathrm{t}}$

The position of bat depends on current velocity of the bat, and then the $X_{\text {new }}$ is its updated position, where $£$ is a random number with uniform distribution $[-1,1]$.

\section{Demand Response System Model}

Let us consider a demand response system consists of one energy provider and n number of users (Fig. 1). Every user has a smart power meter with a built in energy consumption controller (ECC) [10]. The main role of ECC is to control usage of power for every user. The intended time cycle for the system operation is divided into $\mathrm{K}$ time slots, (i.e.) $\mathrm{K}|\kappa|$ where $\kappa$ is the set of all time slots [6]. This division is based on the behavior of user and power demand pattern. The time slots are divided into ON, OFF, and MID slots. Similarly the energy requirement of $\mathrm{N}$ users can be defined as $E\left(E_{1}, E_{2} \ldots E_{n}\right)$. The usage of energy for each user is unpredictable, but somehow the trend during the three slots can be estimated. However machine learning and stochastic signal processing technique can be adopted in each user determine its required demand information. The normal pattern of user's power consumption can fed into appropriate machine learning algorithm to extract the demand information of the users. In order to provide to provide required energy of user $n$ is

$\Sigma_{K \in k}^{n} \mathrm{x}^{\mathrm{k}} \geq \mathrm{E}_{\mathrm{n}}$

Here $\mathrm{x}^{\mathrm{k}}$ is power consumption level and $\mathrm{E}_{\mathrm{n}}$ is energy requirement level for $n$ number of users. In each time slot the power can be classified into maximum and minimum value as $M_{n}$ and $m_{n}$ respectively. Total energy requirement is whereas this tends to be a problem for energy provider 
to find out demand information of all users. Depending on Price the loads can be classified into Price inelastic and elastic loads. Price inelastic loads are loads with fixed consumption level e.g. Refrigerator which provides energy for all time. There is no way of adjusting loads. While elastic load are flexible loads e.g. Charging equipment's, AC etc. [9]. Pre-determined time slot and consumption level can be assigned for this load task, it will be very helpful in power consumption. In this paper Price elastic loads are considered.

\section{Utility Function and Cost Function}

Utility function serves as intermediate action to participate in multiple whole sale market, including day ahead, real time balancing to provide enough energy to meet demand of $\mathrm{N}$ users. In paper [7] each appliance load is calculated and power for time $t$ is drawn out. For each appliance a $\epsilon$ $\mathrm{A}_{\mathrm{i}}$ of customer $\mathrm{i}$, we denote $\mathrm{q}_{\mathrm{i}, \mathrm{a}}(\mathrm{t}) \epsilon \mathrm{t}$ of power drawn in 24 hours. The cost are always kept on increasing with respect to available energy i.e. cost function are strictly convex. Many researchers are developing algorithms to satisfy the demand response of the user's and also to reduce the cost function.

\section{Model Formulation}

Let $l_{1}, l_{2} \ldots . . l_{n}$ and $\mathrm{w}_{1}, \mathrm{w}_{2} \ldots \mathrm{w}_{\mathrm{n}}$ represent elements of the learning vector and their associated weights respectively. [8]Therefore, we define the vector $L_{v}$ as learning vector as follows

$\mathrm{L}_{\mathrm{v}}=\left(l_{1}, l_{2} \ldots l_{n} ; \mathrm{w}_{1}, \mathrm{w}_{2} \ldots \mathrm{w}_{\mathrm{n}}\right)$

Here $\mathrm{w}_{1}$ to $\mathrm{w}_{\mathrm{n}}$ are weights associated with learning vectors $l_{1}$ to $l_{n}$. These adaptive vectors tend to adapt with schedules taking place in system, they are represented as $\mathrm{a}_{1} \mathrm{a}_{2} \ldots \mathrm{a}_{\mathrm{k}}$. Here depending upon the power usage of user the vectors adapt to it. Let $\hat{A}$ be corresponding adaptive vector values as follows

$\hat{\mathrm{A}}=f \mathrm{~A}_{\mathrm{v} 1}, \mathrm{~A}_{\mathrm{v} 2 \ldots . . .} \mathrm{A}_{\mathrm{vn}} g$

$\bar{L}_{\mathrm{v}}=f \mathrm{~L}_{\mathrm{v} 1}, \mathrm{~L}_{\mathrm{v} 2} \ldots . . . \mathrm{L}_{\mathrm{vn}} g$

Here $\left\{A_{v 1}, A_{v 2} \ldots . . A_{v n}\right\}$ and $\left\{L_{v 1}, L_{v 2} \ldots . . L_{v n}\right\}$ shows set of adaptive vectors and its learning vector basis of power usage.

\section{Applying Adaptive Fuzzy Logic Algorithm with Bat Algorithm}

Firstly the algorithm captures number of users. Random vector frequency and vector rate of emission for learning vectors is assigned to reach their destination. The number of learning vectors are taken, the frequency rate of emission and loudness are assigned randomly. With help of these conditions algorithm is continued, if any missing or interruption occurs in between then condition is terminated. Condition is stopped when they are not fulfilled and return back to initial condition. Then learning vector $L_{v}(6)$ with update of velocity and location changes into adaptive vector, it helps in calculating power usage of each user. These vectors travel passes through communication layer to every user using Eqs. 1 and 2. Here minimum and maximum frequency levels are assigned randomly Energy provider provides random range of frequency from $f_{\max }$ and $f_{\min }$. When vectors reach the user it identifies distance, velocity and frequency for the vector for communication between ECC in smart meter and energy provider. This new values are recorded for further use. The main benefit of going for this algorithm is the signal finds out the optimized path of transmitting signals is extracted through this algorithm. Once vector obtain its new location and velocities then new path is obtained $\mathrm{x}_{\text {new }}$. By which velocity and location is updated after it reaches the destination. The updated velocity is calculated using Eq. 3 and power consumption (7). These set of vectors which are generated in each 24 hours are helpful for energy provider to predict the usage rate for future use. $\mathrm{x}_{\text {new }}$ is the new path obtained from adaptive vector. These vectors are further processed by transmitting randomly $x_{\text {rand }}$. if $x_{\text {rand }}$ is lower than rate of emission then local solution is generated and $x_{\text {best }}$ is extracted out. If $\mathrm{x}_{\text {rand }}$ is higher, it is further processed by flying randomly until $x_{\text {new }}$ is lesser than $x_{\text {rand }}$. The simplified form is shown in flowchart Fig. 2.

By varying population size and frequency the algorithm is checked and simulation results are shown in Fig. 3. For each variation $\mathrm{x}_{\text {new }}$ is obtained. When random new solution is less than or equal to pre-defined $X_{\text {best }}$ solution then users consumption level is optimal or else with help these algorithm over usage indication can be send to the user. This flow chart was evaluated as algorithm and executed with simulation code.

\section{Performance Evaluation}

In this section the proposed algorithm is simulated and results are shown in Fig. 3. Here the usage of single user is evaluated. Power usage (in $\mathrm{kW}$ ) vs time (in hours) here schedule of 24 hours is taken from 12 a.m to 12 a.m of next day are evaluated. In simulation results, power hike is observed in between 5 to 10 a.m. the path obtained through this algorithm transmits information to energy provider, and he transmits information to user about power usage. 
Fig. 2 Flow chart for the proposed system

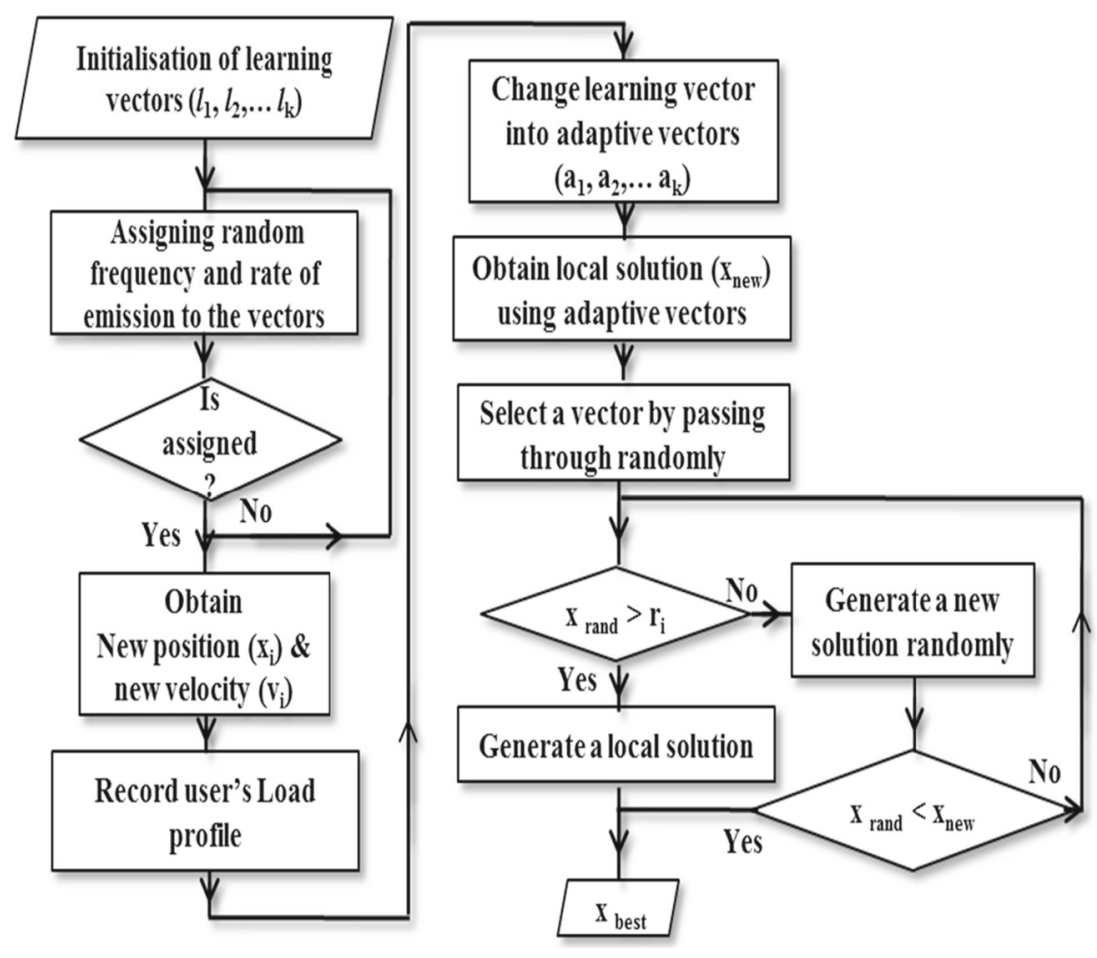

To reduce the cost function, the users will also participate in demand side to get better results. This algorithm helps in prediction of power usage of every single user. So that user will also be aware of their power consumption. In order to save power during peak time the user/energy provider can take decision to change power source from non-renewable resource to renewable energy source like solar, wind etc., that will help in saving major amount non-renewable resource. For e.g. between 5-10 am and 4-6pm, if the solar power panel with battery acts as power source for a user then major amount of non-renewable resource can be saved.

\section{Conclusion}

This paper presented a new approach of adaptive fuzzy logic system in smart grid using bat algorithm. Here offline algorithm is generated and applied in Matlab 2016a and random values of input are given as frequency and emission rate for vectors and simulation is evaluated as shown in Fig. 3. By which 24 hours power consumption single user schedule is listed out. On comparing to other algorithm these proposed algorithm is helpful in communicating with the user through smart meter to reduce the power
Fig. 3 Simulated smart grid power usage in 24 hours of time

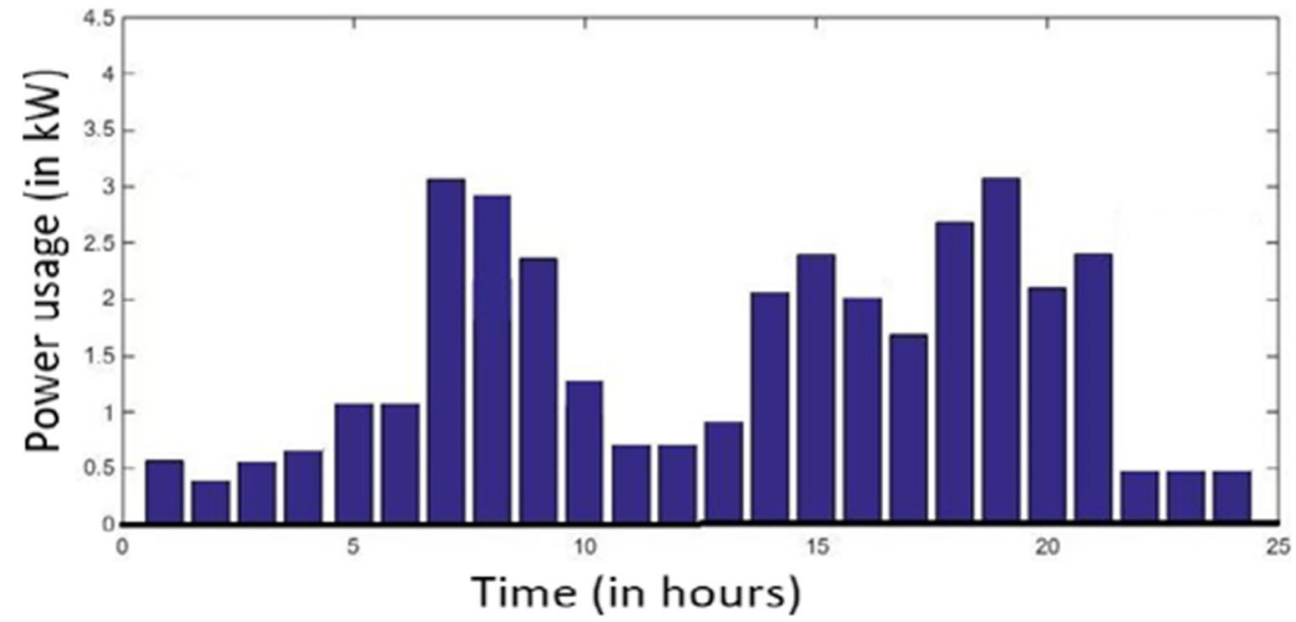


consumption whenever there is peak in power takes place. The algorithm is designed in way such that adaptive vectors make use of bat algorithm to find the optimal path of information flow and also gets communicated to user from energy provider. Then it starts to analyze day to day power usage of user and when there is peak in load the indication is given to user and also helps energy provider in predicting for every single user demand through this algorithm.

Acknowledgments The authors would like to express their sincere thanks to Dr. S R K Prasad (Correspondent, CIT), Dr. R. Prabhakar (Secretary, CIT), Dr. V. Selladurai (Principal, CIT), and Mr. P. Rajiv Rangasamy (Director, CIT) for their help and Encouragement during the course of work. The authors like to thank Dr. Hongbo Liu, Professor Eje Central Lazaro Cardenas, Mexico for useful discussion.

\section{References}

1. Momoh J (2012) Text book on 'Smart grid Fundamentals of Design and Analysis', 1st edn. Wiley-IEEE Press, New York

2. RaisulAlam M, St-Hilaire M, Kunz T (2013) 'A survey on cost saving methods for smart grids' Published in IEEE International Conference on Smart Energy Grid Engineering (SEGE'13). https://doi.org/10.1109/TII.2015.2414719

3. MiroslawLazoryszczak JPP The stability analysis of Bat Algorithm. In: 2017 IEEE international conference on innovations in intelligent systems and applications (INISTA2017). https://doi. org/10.1109/INISTA.2017.8001133

4. SankalapArori SS A Conceptual Comparison of Firefly Algorithm, Bat Algorithm and Cuckoo Search. In: 2013 international conference on control, computing, communication and materials (ICCCCM). https://doi.org/10.1109/ICCCCM.2013.6648902

5. Yang X-S (2010) A new metaheuristic bat-inspired algorithm. In: Nature inspired cooperative strategies for optimization (NICSO 2010). Springer, pp 65-74. https://doi.org/10.1007/978-3-64212538-6_6

6. Samadi P, Member S, Mohsenian-Rad H, Schober R, Wong VWS (2012) Advanced Demand Side Management for the Future Smart Grid Using Mechanism Design. IEEE Trans Smart Grid 3(3):1170-1180. https://doi.org/10.1109/TSG.2012.2203341

7. Li N, Chen L, Steven H (2011) Optimal demand Response Based on Utility Maximization in Power Networks. In: Power and energy society general meeting. https://doi.org/10.1109/PES. 2011.6039082

8. Siamakarzanpour A (2017) 'An adaptive fuzzy logic system for residential energy management in smart grid environment fuzzy logic system for residential energy management in smart grid environments' Applied Energy (2016), Volume 186, Part 1, pp 68-81. https://doi.org/10.1016/j.apenergy.2016.11.028

9. Bahrami S, Wong WS, Huang J An Online Learning Algorithm for Demand Response in Smart Grid. IEEE Trans Smart Grid PP(99):4712-4725. https://doi.org/10.1109/TSG.2017.2667599

10. Al-ali AR, Tubaiz NA, Al-Radaideh A, Al-Dmour JA, Murugan L (2012) Smart Grid Controller for Optimizing HVAC Energy Consumption. In: 2012 International conference on computer systems and industrial informatics (ICCSII). https://doi.org/10. 1109/ICCSII.2012.6454548 